\title{
EFECTIVIDAD DE LA MEZCLA PICLORAM Y FLUROXIPIR EN EL CONTROL DE MALEZAS PERENNES DE PASTIZALES TROPICALES ${ }^{1}$
}

\author{
Valentín A. Esqueda Esquivel ${ }^{2}$, Oscar H. Tosquy Valle ${ }^{2}$, Enrique Rosales Robles ${ }^{3}$
}

\begin{abstract}
RESUMEN
Efectividad de la mezcla picloram y fluroxipir en el control de malezas perennes de pastizales tropicales. $\mathrm{Du}$ rante el año 2001 se estableció un experimento en tres localidades del centro y norte del estado de Veracruz, México, con el objeto de conocer la efectividad de la mezcla formulada de los herbicidas picloram + fluorxipir en el control de escobilla (Sida acuta Burm f.), olín (Croton reflexifolius Kunth) y puzgual (C. cortesianus Kunth), tres especies importantes de malezas perennes de pastizales tropicales. En diseño experimental de bloques completos al azar con cuatro repeticiones, se evaluaron las mezclas de picloram + fluroxipir a $40+40,60$ +60 y $80+80$ g i. a. $/ 1001$ de agua; picloram $+2,4-\mathrm{D}$ a $64+$ 240 y $96+360 \mathrm{~g} / 1001$ de agua y picloram + metsulfurón metil a $64+4 \mathrm{~g} / 1001$ de agua. Se determinó el control por los tratamientos sobre las tres malezas y su toxicidad a los pastos. Los mejores controles de escobilla y puzgual se obtuvieron con las dosis alta e intermedia de picloram + fluroxipir, con valores promedio superiores a $90 \%$. Esta mezcla superó significativamente en control a los tratamientos de picloram $+2,4-$ D y picloram + metsulfurón metil. Con las dosis altas de picloram + fluroxipir y de picloram + 2,4-D, se obtuvieron controles de olín de 55 y $46 \%$, respectivamente; estas mezclas fueron estadísticamente semejantes entre sí. Los tratamientos herbicidas no provocaron daños de toxicidad a los pastos. Se concluye que para tener un buen control de escobilla y puzgual, con la mezcla de picloram + fluroxipir se requiere aplicar al menos la dosis de $60+60 \mathrm{~g}$ i. a. $/ 100 \mathrm{l}$ de agua.
\end{abstract}

Palabras clave: Sida acuta, Croton reflexifolius, Croton cortesianus, control químico, 2,4-D, metsulfurón metil.

\begin{abstract}
Effectiveness of the mixture picloram and fluroxypyr on the control of perennial weeds of tropical grasslands. During 2001 an experiment was established in three locations of the central and northern areas of the state of Veracruz, Mexico. The objective was to assess the effectiveness of the formulated mixture of the herbicides picloram + fluorxypyr in the control of Sida acuta Burm f., Croton reflexifolius Kunth and $C$. cortesianus Kunth, three perennial weed species common in tropical grasslands. The mixtures of picloram + fluroxypyr at $40+40,60+60$ and $80+80 \mathrm{~g}$ a. i./100 1 water; picloram $+2,4-\mathrm{D}$ at $64+240$ and $96+360 \mathrm{~g} / 100 \mathrm{l}$ water and picloram + metsulfuron methyl at $64+4 \mathrm{~g} / 1001$ water were evaluated in a Randomized Complete Block Design with four replications. The effect of the treatments on the control of the three weed species and on the toxicity to the forage grasses was determined. The best control of $S$. acuta and $C$. cortesianus were obtained with the highest and medium rates of picloram + fluroxypyr, with average values higher than $90 \%$. This herbicide mixture had a significantly higher control than the picloram $+2,4-\mathrm{D}$ and picloram + metsulfuron methyl treatments. With the highest rates of picloram + fluroxypyr and picloram +2,4-D, C. reflexifolius was controlled 55 and 46\%, respectively; these mixtures were statistically similar between them. None of the herbicide treatments caused any toxicity to the forage grasses. It was concluded that applications of at least $60+60 \mathrm{~g}$ a. i./ha. of picloram + fluroxypyr are required to have a good control of $S$. acuta and C. cortesianus.
\end{abstract}

Key words: Sida acuta, Croton reflexifolius, Croton cortesianus, chemical control, 2,4-D, metsulfuron methyl.

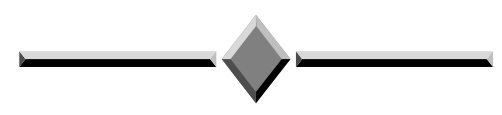

1 Recibido: 2 de junio, 2005. Aceptado: 30 de agosto, 2005.

2 Campo Experimental Cotaxtla. INIFAP. Km. 34 Carr. Veracruz-Córdoba. Apdo. Postal 429, 91700, Veracruz, Ver., México. Correo electrónico: esqueda.valentin@inifap.gob.mx; tosquy.oscar@inifap.gob.mx

3 Campo Experimental Río Bravo. INIFAP. Km. 61 Carretera Matamoros-Reynosa.Apdo. Postal 172, 88900, Río Bravo, Tamps., México. Correo electrónico: rosales.enrique@inifap.gob.mx 


\section{INTRODUCCIÓN}

En el estado de Veracruz se dedican cerca de 3,6 millones de hectáreas al pastoreo de ganado vacuno, que representan alrededor de $50 \%$ de la superficie estatal; el estado es el primer productor nacional de carne de bovino y el quinto en producción de leche (Juárez et al. 2000).

Uno de los principales factores que afectan la producción y la calidad de los pastos en los agostaderos, es la presencia de maleza, principalmente de especies de hoja ancha, ya que éstas compiten fuertemente con los pastos por agua, luz y nutrimentos, reducen la superficie de pastoreo aprovechable, son refugio de fauna nociva, como roedores y reptiles y pueden ser tóxicas o causar lesiones al ganado (Avila 1988; Silva et al. 1990). Debido a que en Veracruz, los potreros se encuentran en una gran diversidad de ambientes edáficos y climáticos, existe un amplio número de especies de malezas de hoja ancha, tanto herbáceas, como arbustivas (Enríquez et al. 1999). De estas últimas, hay alrededor de 50 especies entre las que destacan el puzgual (Croton cortesianus Kunth) y el olín (C. reflexifolius Kunth), especies de la familia Euphorbiaceae que se presentan principalmente en la zona norte del estado (Reyes 1970). A su vez, la escobilla (Sida acuta Burm f.), una planta semiarbustiva de la familia Malvaceae que se presenta sobre todo en el centro y sur del estado, es catalogada como una especie de difícil control con los herbicidas actualmente utilizados.

El control de las malezas en los potreros se realiza mediante chapeos manuales y mecánicos o con la aplicación de herbicidas selectivos (Hernández y Reichert 1987). Con los chapeos, solamente se logra un control temporal de las malezas (Radillo y Nava 2001), por lo que deben repetirse periódicamente durante la época de lluvias (Reichert 1986). A su vez, con el control químico se puede lograr la eliminación gradual de las malezas, ya que de un ciclo a otro, se disminuye significativamente su población y por tanto, los costos relacionados a su control (Motooka 1986; Esqueda 2000b). Sin embargo, existe escasa información sobre estos aspectos, por lo que es necesario desarrollar tecnología sobre el control de malezas en los pastizales de Veracruz.

Actualmente, el control químico de malezas más utilizado en los potreros del estado, consiste en la aplicación en diferentes dosis de la mezcla formulada de picloram + 2,4-D y en menor proporción se utiliza la mezcla de picloram + metsulfurón metil. Cada mezcla tiene un espectro de especies herbáceas y arbustivas que controla, pero su efecto es limitado en algunas especies importantes de maleza, sobre todo arbustivas o semiarbustivas, como las mencionadas anteriormente (Reichert 1989). Con base en resultados de evaluaciones preliminares, se determinó que la mezcla formulada de los herbicidas picloram + fluroxipir, puede ser una nueva alternativa de control para algunas de las malezas de importancia en los potreros (Esqueda 2000a; Reichert 1998). Por lo anterior, el objetivo de este trabajo fue determinar la efectividad de esta mezcla a diferentes dosis en el control de las especies antes mencionadas y compararla con mezclas herbicidas que se emplean en la actualidad.

\section{MATERIALES Y MÉTODOS}

Durante el mes de marzo de 2001 se estableció un experimento de mezclas herbicidas en pastizales de tres localidades del estado de Veracruz, donde prevalecían poblaciones representativas de escobilla, olín y puzgual. Para escobilla, el sitio experimental se ubicó en un potrero con gramas nativas en el Ejido Juan de Alfaro, municipio de Medellín de Bravo, en el centro del estado. Para olín se utilizó un pastizal sembrado con los zacates Pangola (Digitaria decumbens Stent.) y Estrella (Cynodon plectostachyus Pilger), en el Rancho El Trancazo, en el poblado de El Chote, municipio de Papantla, en la zona norte, y para puzgual el sitio seleccionado fue un pastizal sembrado con zacate Pangola, ubicado en el Rancho Chapopote Núñez, en el poblado Chapopote, municipio de Alamo, también en el norte del estado. El diseño experimental utilizado fue de bloques completos al azar con cuatro repeticiones. Las dimensiones de las parcelas experimentales fueron de cinco $\mathrm{m}$ de largo $\mathrm{x}$ cuatro $\mathrm{m}$ de ancho $\left(20 \mathrm{~m}^{2}\right)$.

Se evaluaron seis tratamientos conformados de la siguiente manera: mezcla formulada de picloram + fluroxipir aplicada en tres dosis; mezcla formulada de picloram + 2,4-D aplicada en dos dosis; mezcla de tanque de picloram + metsulfurón metil aplicada en una dosis y un testigo sin aplicación (Cuadro 1).

En los lotes experimentales del Ejido Juan de Alfaro y del Rancho El Trancazo, los tratamientos se aplicaron el 30 de marzo de 2001; a su vez, los tratamientos del experimento localizado en el Rancho Chapopote Núñez, se aplicaron el 1 de abril del mismo año. Para la aplicación de los tratamientos, se utilizó una aspersora de mochila de acción manual, equipada con una boquilla Lurmark 04-F110. Cada mezcla herbicida se asperjó a manera de cubrir completamente las malezas presentes en cada una de las parcelas experimentales, pero sin llegar al escurrimiento. Al momento de las aplicaciones, la población de escobilla era de 222.500 plantas/ha y su altura promedio de $23,5 \mathrm{~cm}$; la población de 
Cuadro 1. Descripción de los tratamientos para el control de maleza en pastizales. Veracruz, México. 2001.

\begin{tabular}{llc}
\hline No. & \multicolumn{1}{c}{ Tratamientos } & $\begin{array}{c}\text { Dosis } \\
\text { (g i. a./100 I agua) }\end{array}$ \\
\hline & & \\
1 & Picloram + fluroxipir & $40+40$ \\
2 & Picloram + fluroxipir & $60+60$ \\
3 & Picloram + fluroxipir & $80+80$ \\
4 & Picloram + 2,4-D & $64+240$ \\
5 & Picloram + 2,4-D & $96+360$ \\
6 & Picloram + metsulfurón metil $^{1}$ & $64+4$ \\
\hline
\end{tabular}

1 Tratamientos utilizados como testigos regionales de control químico.

puzgual de 25.000 plantas/ha, con altura promedio de $40,5 \mathrm{~cm}$ y la población de olín era de 16.250 plantas/ha, con una altura promedio de $69,7 \mathrm{~cm}$. Tanto la escobilla, como el puzgual y el olín eran las especies dominantes por población o cobertura en sus respectivos lotes experimentales. En las parcelas correspondientes a los testigos sin aplicar, se permitió a las malezas desarrollarse libremente durante el período de conducción de los experimentos.

Como variables de respuesta se consideraron el control de malezas y la toxicidad causada por los herbicidas a los pastos.

\section{Control de malezas}

Las evaluaciones de control de escobilla y puzgual, se llevaron a cabo a los 15,30 y 60 días después de la aplicación de los tratamientos (DDA), mientras que las de olín, se efectuaron a los 30, 90 y 180 DDA. Se describieron los síntomas ocasionados por los tratamientos herbicidas en las plantas de escobilla, olín y puzgual tratadas y se evaluó visualmente su control. En todos los casos se utilizó la escala de 0 a $100 \%$, en donde 0 significó que las malas hierbas no fueron afectadas y $100 \%$ que fueron completamente destruidas (Esqueda 2003).

\section{Toxicidad a los pastos}

En las mismas fechas de evaluación de control de maleza, se realizó una evaluación sobre los pastos, para determinar si los tratamientos ocasionaron daños fitotóxicos a los mismos. Se utilizó la escala de 0 a $100 \%$, en donde 0 significó que los pastos no fueron dañados y $100 \%$ que fueron eliminados en su totalidad (Esqueda 2003).

\section{Análisis estadísticos}

Para homogenizar las varianzas, los datos experimentales de control de las tres especies de maleza fueron transformados a su valor de arco seno multiplicado por la raíz cuadrada del porcentaje (Gomez y Gomez 1984). Se realizaron análisis de varianza por especie de maleza y fecha de evaluación y donde se detectó significancia se aplicó la prueba de Diferencia Mínima Significativa (DMS) al 0,05 de probabilidad de error. También se realizaron contrastes ortogonales en el control final de cada especie, para comparar la eficiencia promedio de la nueva mezcla picloram + fluroxipir con las mezclas picloram + 2,4-D $(\mathrm{T} 1+\mathrm{T} 2+\mathrm{T} 3 / 3$ versus $\mathrm{T} 4+\mathrm{T} 5 / 2)$ y picloram + metsulfurón metil ( $\mathrm{T} 1+\mathrm{T} 2+\mathrm{T} 3 / 3$ versus $\mathrm{T} 6)$, así como entre las tres dosis de la nueva mezcla (T1 versus T2; T2 versus T3 y T1 versus T3). Aún cuando los análisis estadísticos se efectuaron con datos transformados, por motivos de claridad, la información de control de malezas se presenta con los datos reales.

\section{RESULTADOS Y DISCUSIÓN}

\section{Síntomas de toxicidad en las malezas}

El efecto fitotóxico ocasionado por todas las mezclas herbicidas en las tres especies de malezas, se manifestó inicialmente como una clorosis ligera en las hojas, la cual aumentó progresivamente hasta adquirir una coloración café-naranja, para posteriormente llegar a una necrosis parcial o total del follaje; la intensidad de estos síntomas fue directamente proporcional a la dosis aplicada.

\section{Control de escobilla}

Los resultados para esta especie se muestran en el Cuadro 2, donde se observa que a los 15 DDA, picloram + fluroxipir a su dosis más alta mostró un $80 \%$ de control, el cual fue estadísticamente superior al obtenido con el resto de los tratamientos. Las dosis intermedia y baja de esta mezcla tuvieron un control similar al ofrecido por la dosis alta de picloram $+2,4-\mathrm{D}$ y picloram + metsulfurón metil. Con las dosis bajas de picloram + 2,4-D y picloram + fluroxipir, se tuvieron los menores controles de esta especie (50 y 60\%, respectivamente). $\mathrm{A}$ los $30 \mathrm{DDA}$, los tratamientos incrementaron el control de escobilla entre 7 y $16 \%$, aunque éstos guardaron el mismo comportamiento estadístico. A los 60 DDA, los mejores controles de escobilla (93 y $90 \%$ ), se obtuvieron con las dosis alta e intermedia de picloram + fluroxipir, respectivamente. El control de esta maleza con las dosis de los tratamientos testigos, fue estadísticamente semejante al de la dosis baja de picloram + fluroxipir. 
Cuadro 2. Control de escobilla a los 15, 30 y 60 días después de la aplicación de los tratamientos (DDA). Veracruz, México. 2001.

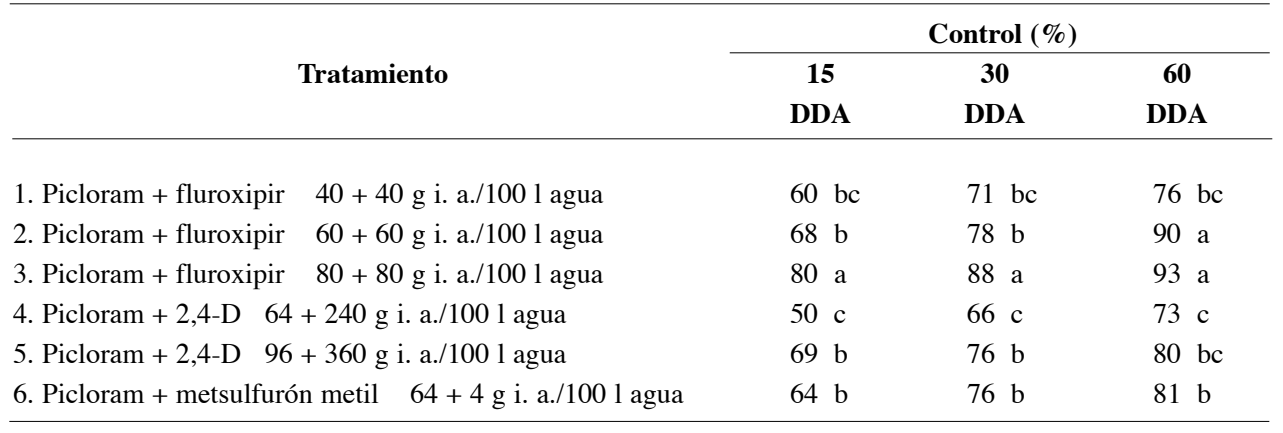

Cifras con letras iguales dentro de columnas no difieren estadísticamente (DMS 0,05).

El análisis de contrastes determinó que para controlar escobilla, picloram + fluroxipir es significativamente mejor que picloram $+2,4-\mathrm{D}$ y picloram + metsulfurón metil, proporcionando controles promedio 10,3 y $5,5 \%$ superiores que los obtenidos con las mezclas testigo. Asimismo, se confirmó que se tiene un control de escobilla semejante al aplicar las dosis alta e intermedia de picloram + fluroxipir, y que ambas dosis controlan esta especie, mejor que la dosis baja.

Estos resultados concuerdan con Reichert (1998) y Esqueda (2000a) y ponen de manifiesto que existen otras alternativas para el control químico de escobilla que superan significativamente a las de actual uso.

\section{Control de olín}

A los 30 DDA, los mejores resultados se obtuvieron con las dosis altas de picloram $+2,4$-D y picloram + fluroxipir, aunque su control estuvo por debajo de
70\%. A los 90 DDA, ambos tratamientos conservaron estadísticamente los mayores porcentajes de control de olín, no obstante que se observó, una reducción en el efecto de la mezcla de picloram + 2,4-D. En la última fecha de evaluación, el mejor tratamiento fue la dosis alta de picloram + fluroxipir con 55\% de control. Por su parte, los tratamientos mas deficientes en el control de esta maleza, fueron la dosis baja de la mezcla picloram + fluroxipir y la mezcla testigo de picloram + metsulfurón metil (Cuadro 3).

Cabe destacar, que el efecto de las mezclas herbicidas disminuyó después de los 30 DDA, excepto en los tratamientos con la dosis más alta de picloram + fluroxipir y de picloram + metsulfurón metil, en los cuales su control disminuyó hasta después de los 90 DDA.

El análisis de contrastes corroboró que en el control de olín, la dosis alta de la mezcla de picloram + fluroxipir fue significativamente superior que las dosis intermedia y baja, en 17,5 y $28,75 \%$, respectivamente. No

Cuadro 3. Control de olín a los 30, 90 y 180 días después de la aplicación de los tratamientos (DDA). Veracruz, México. 2001.

\begin{tabular}{|c|c|c|c|c|}
\hline \multirow{3}{*}{\multicolumn{2}{|c|}{ Tratamiento }} & \multicolumn{3}{|c|}{ Control (\%) } \\
\hline & & \multirow{2}{*}{$\begin{array}{c}\text { 30 } \\
\text { DDA }\end{array}$} & \multirow{2}{*}{$\begin{array}{c}90 \\
\text { DDA }\end{array}$} & \multirow{2}{*}{$\begin{array}{c}180 \\
\text { DDA }\end{array}$} \\
\hline & & & & \\
\hline 1. Picloram + fluroxipir & $40+40$ g i. a. $/ 1001$ agua & $44 \mathrm{c}$ & $36 \mathrm{~d}$ & $26 \mathrm{~d}$ \\
\hline 2. Picloram + fluroxipir & $60+60$ g i. a./100 l agua & $55 \mathrm{~b}$ & 50 bcd & $38 \mathrm{c}$ \\
\hline 3. Picloram + fluroxipir & $80+80$ g i. a./100 l agua & 66 a & 68 a & 55 a \\
\hline 4. Picloram + 2,4-D 6 & $64+240$ g i. a./100 l agua & $58 \mathrm{~b}$ & $51 \mathrm{bc}$ & $39 \mathrm{bc}$ \\
\hline 5. Picloram + 2,4-D & $96+360$ g i. a./100 1 agua & $69 \mathrm{a}$ & $60 \mathrm{ab}$ & $46 \mathrm{~b}$ \\
\hline 6. Picloram + metsulfur & rón metil $64+4 \mathrm{~g}$ i. a./100 1 agua & $40 \mathrm{c}$ & $48 \mathrm{~cd}$ & $34 \mathrm{~cd}$ \\
\hline
\end{tabular}

Cifras con letras iguales dentro de columnas no difieren estadísticamente (DMS 0,05). 
se detectó efecto significativo al comparar el valor promedio de control de las tres dosis de la mezcla de picloram + fluroxipir, con el valor promedio de las dos dosis de la mezcla de picloram $+2,4-\mathrm{D}$ y la mezcla de picloram + metsulfurón metil.

El efecto de los tratamientos en el olín fue de mediano a bajo, lo cual se debe a sus características de reproducción por rizomas y a la consistencia coreácea y cerosa que tienen sus hojas. Esto señala la necesidad de realizar más investigación en el control de esta especie.

\section{Control de puzgual}

A los 15 DDA, la dosis alta de picloram + fluroxipir mostró un control de puzgual mayor a $90 \%$, estadísticamente superior al resto de los tratamientos. Le siguieron en importancia la dosis intermedia y baja de esta misma mezcla. El control de esta especie con la dosis alta de picloram $+2,4-\mathrm{D}$ fue similar al obtenido con la dosis baja del mismo tratamiento y superior a la mezcla de picloram + metsulfurón metil. A los 30 DDA, todas las mezclas herbicidas incrementaron el porcentaje de control de esta maleza, excepto picloram + metsulfurón metil. La dosis alta de picloram + fluroxipir se mantuvo como el mejor tratamiento, seguido de la misma mezcla en dosis intermedia. A los 60 DDA, se observó un ligero incremento en el control de puzgual con todos los tratamientos herbicidas, excepto con la dosis alta de picloram + 2,4-D. El mejor control final de esta especie se obtuvo con la dosis alta de picloram + fluroxipir, seguido por la dosis intermedia de la misma mezcla (Cuadro 4).

La comparación de promedios mediante contrastes, indicó que para el control de puzgual, la mezcla de picloram + fluroxipir fue superior en 26,5 y $60,25 \%$, a las mezclas testigo picloram $+2,4$-D y picloram + metsulfurón metil, respectivamente. Asimismo, la dosis alta de picloram + fluroxipir, superó significativamente a las dosis intermedia y baja de esta mezcla.

\section{Toxicidad a los pastos}

En ninguno de los experimentos, los tratamientos evaluados provocaron síntomas de toxicidad en los pastos sobre los que fueron aplicados (datos no mostrados). Con lo anterior, se demuestra que la nueva mezcla formulada de picloram + fluroxipir, puede utilizarse sin riesgo de provocar daños fitotóxicos a los pastos Pangola, Estrella de África y gramas nativas.

Los resultados de este estudio permiten determinar, que ninguno de los tratamientos estudiados controla completamente a las tres especies de maleza, por lo que, la selección de un tratamiento para su control químico debe basarse en las especies de maleza presentes en los pastizales.

Con base en lo anterior, se concluye que para obtener un buen control de escobilla con la nueva mezcla de picloram + fluroxipir, se requiere aplicarla en dosis de al menos $60+60 \mathrm{~g}$ i. a. por 100 litros de agua. Para puzgual, aunque estadísticamente la mejor opción fue la aplicación de $80+80 \mathrm{~g}$ i. a. de picloram + fluroxipir por 100 litros de agua, en forma práctica, es suficiente aplicar $60+60$ g i. a. por 100 litros de agua. Esta combinación ofrece un control más eficiente de estas especies que el que se obtiene con los herbicidas que actualmente se utilizan en los pastizales del estado de Veracruz. Ninguno de los tratamientos proporcionó un control comercialmente satisfactorio del olín.

Cuadro 4. Control de puzgual a los 15, 30 y 60 días después de la aplicación de los tratamientos (DDA). Veracruz, México. 2001.

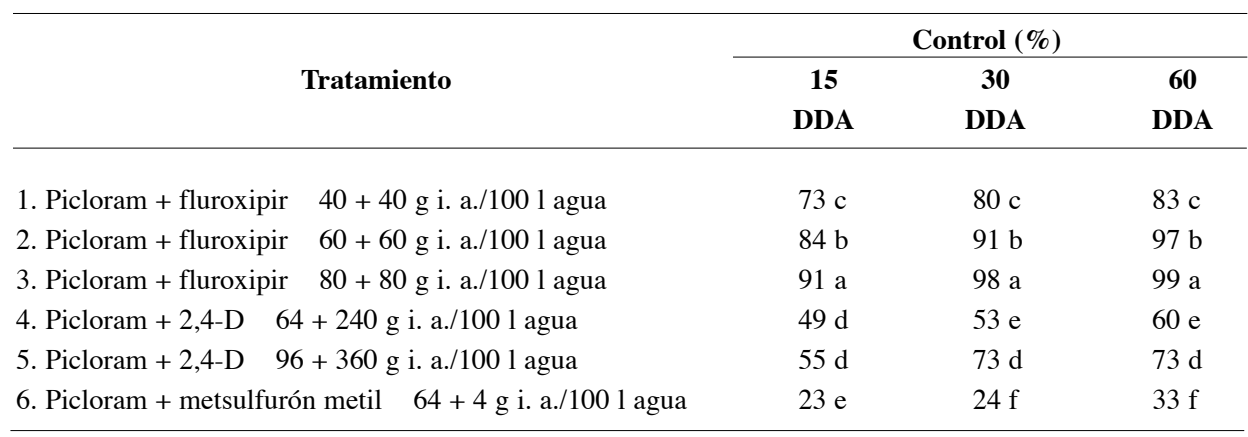

Cifras con letras iguales dentro de columnas no difieren estadísticamente (DMS 0,05). 


\section{LITERATURA CITADA}

AVILA, J. M. 1988. Efecto de dos herbicidas y diesel sobre el control de mezquite (Prosopis juliflora) y huizache (Acacia farnesiana). In: SOMECIMA ed. Memorias IX Congreso Nacional de la Ciencia de la Maleza. Cd. Juárez, Chihuahua, México. p. 231-236.

ENRÍQUEZ, J. F.; MELÉNDEZ, F.; BOLAÑOS, E. D. 1999. Tecnología para la producción y manejo de forrajes tropicales en México. INIFAP. CIRGOC. Campo Experimental Papaloapan. Libro Técnico Núm. 7. División Pecuaria. México. 262 p.

ESQUEDA, V. A. 2000a. Efecto del chapeo manual y dos herbicidas en el control de la maleza y la producción de forraje de zacate Pangola (Digitaria decumbens Stent.). In: Fefer, F. ed. Memorias XXI Congreso Nacional de la Ciencia de la Maleza. Morelia, Michoacán, México. p. 112-117.

ESQUEDA, V. A. 2000b. Evaluación de la producción de forrajes tropicales con diversos controles de malezas. In: Barradas, H. ed. Memorias XIII Reunión Científica Tecnológica Forestal y Agropecuaria Veracruz 2000. Veracruz, Veracruz, México. s.p.

ESQUEDA, V. 2003. Evaluación de los herbicidas Crosser y Vaquero en el control de malezas herbáceas en pastizales tropicales. Agronomía Mesoamericana 14(2):177-183.

GOMEZ, K. A.; GOMEZ, A. A. 1984. Statistical Procedures for Agricultural Research. 2nd ed. New York, USA. Wiley. 680 p.

HERNÁNDEZ, J. O.; REICHERT, A. 1987. Evaluación de 5 herbicidas sobre el control de malezas en potreros de clima Af (c). In: SOMECIMA ed. Memorias VIII Congreso Nacional de la Ciencia de la Maleza. San Luis Potosí, San Luis Potosí, México. p. 123-127.

JUÁREZ, F. ; CONTRERAS, J.; MONTERO, M. 2000. Determinación de la tasa de digestión de gramíneas tropicales en el estado de Veracruz. In: Barradas, H. ed. Memorias XIII Reunión Científica Tecnológica Forestal y Agropecuaria Veracruz 2000. Veracruz, Veracruz, México. s.p.
MOTOOKA, P. S. 1986. Chemical weed control in tropical pastures. In: Moody, K. ed. Weed control in tropical crops. Vol. II. Los Baños Laguna, Philippines: Weed science society of the Philippines. Southeast asian region center for graduate study and research in agriculture. p. 9-54.

RADILLO, J. F.; NAVA, B. 2001. Evaluación de aplicación química y método de chapeo para control de Acacia farnesiana L. Willd en praderas. In: ASOMECIMA ed. Memoria XXII Congreso Nacional de la Ciencia de la Maleza. Colima, Colima, México. p. 56.

REICHERT, A. 1986. El huisache de la parte central de Veracruz problemática y control. In: SOMECIMA ed. Resúmenes VII Congreso Nacional de la Ciencia de la Maleza y VIII Congreso de la Asociación Latinoamericana de Malezas. Guadalajara, Jalisco, México. p. 194.

REICHERT, A. 1989. Evaluación de diferentes herbicidas para el control de olín Croton spp. en potreros de Veracruz. In: ASOMECIMA ed. Resúmenes X Congreso Nacional de la Ciencia de la Maleza. Veracruz, Veracruz, México. p. 25.

REICHERT, A. 1998. Evaluación del herbicida Picloram + Fluroxipir para el control de pusgual (Croton cortesianus Kunth.) y orozús (Lantana camara L.) en áreas ganaderas de Veracruz. In: Fefer, F. ed. Resúmenes XIX Congreso Nacional de la Ciencia de la Maleza. Mexicali, Baja California, México. p. 51.

REYES, T. H. 1970. Evaluación de herbicidas en el control de cinco especies leñosas perjudiciales en potreros del norte y centro del estado de Veracruz. Tesis de Ingeniero Agrónomo. Buenavista, Saltillo, Coahuila, México. Universidad de Coahuila. Escuela Superior de Agricultura Antonio Narro. 45 p.

SILVA; J. H.; BELTRÁN, R. G.; VALDÉZ, M. G. 1990. Estudio preliminar de plantas tóxicas presentes en los agostaderos de la zona norte del estado de Colima. In: SOMECIMA ed. Resúmenes XI Congreso Nacional de la Ciencia de la Maleza. Irapuato, Guanajuato, México. p. 105. 